\title{
On the Ptolemy constant of Lorentz sequence spaces and their duals
}

\author{
Zhan-fei Zuo ${ }^{\mathrm{a}, \mathrm{b}}$ \\ ${ }^{a}$ Department of Mathematics and Statistics, Chongqing Three Gorges University, Wanzhou 404100 China. \\ b Key Laboratory of Intelligent Information Processing and Control, Chongqing Three Gorges University, \\ Wanzhou, Chongqing 404100 China.
}

e-mail: zuozhanfei@163.com

Received 28 Dec 2017

Accepted 13 Jun 2018

\begin{abstract}
The Ptolemy constant of two-dimensional Lorentz sequence spaces is computed in this paper. On the way, we also calculate the Ptolemy constant in the dual space of two-dimensional Lorentz sequence spaces in the case of $q=1$ and $q \geqslant 2$. Moreover, we determine the Ptolemy constant of absolute normalized norms under some new conditions. These results which not only contain some previous results, but also give the exact value of the Ptolemy constant in some concrete Banach spaces.
\end{abstract}

KEYWORDS: Banach spaces; absolute normalized norm; geometric constants

\section{INTRODUCTION}

Let $X$ be a non-trivial Banach space, and $S_{X}=$ $\{x \in X:\|x\|=1\}, B_{X}=\{x \in X:\|x\| \leqslant 1\}$ be the unit sphere of $X$ and unit ball of $X$, respectively. In general, the study of the geometric property of a Banach space $X$ is not easy. Alternatively, one can do this with the help of some geometric constants. Some geometric constants for a Banach space $X$ have been investigated in the literature, such as von Neumann-Jordan constants $C_{N J}(X)^{1-3}$, Zbăganu constant $C_{Z}(X)^{4-6}$, Ptolemy constant $C_{p}(X)^{7-9}$. These constants are important due to its strong connection with some useful geometric properties, such as uniformly nonsquareness and uniform normal structure ${ }^{10-12}$. Moreover, the Ptolemy constant $C_{p}(X)$ turns out to be useful in the study of the equivalence of Green's functions of second-order linear elliptic operators ${ }^{13}$. It is also a useful tool in the study of the existence of positive solutions of certain nonlinear equations ${ }^{14}$. As mentioned above, it is thus meaningful to calculate the exact value of some constants in some concrete spaces $^{15-17}$.

Recently, we give a simple method to determine the Ptolemy constant $C_{p}(X)$ of absolute normalized norms on $\mathbb{R}^{2}$ which are complementary to the results of Llorens-Fuster, Mazcunan-Navarro and Reich ${ }^{7}$. Moreover, the exact values of the Ptolemy constant $C_{p}(X)$ were calculated in some classical Banach spaces, such as the space $\ell_{p}$, Cesàro space $\operatorname{ces}_{p}^{(2)}$, and
Lorentz sequence spaces $d^{(2)}(\omega, 2)^{9,18,19}$. However, to our best knowledge, there are no results about the exact value of Ptolemy constant $C_{p}(X)$ in Lorentz sequence spaces $d^{(2)}(\omega, q)(1 \leqslant q<\infty$ and $q \neq 2)$ and their dual spaces.

In this paper, we firstly determine the exact value of Ptolemy constant $C_{p}(X)$ under some new conditions on the correspondence continuous convex function on $[0,1]$. As an application, we can compute the value of the Ptolemy constant $C_{p}(X)$ in some concrete Banach spaces. Moreover, we completely compute the Ptolemy constant of two-dimensional Lorentz sequence spaces $d^{(2)}(\omega, q)$ $(1 \leqslant q<\infty)$ and their dual spaces $d^{(2)}(\omega, q)^{*}$ in the case of $q=1$ and $q \geqslant 2$. The new results which not only contain some previous results ${ }^{9,20}$, but also give some new results in some concrete Banach spaces.

\section{PRELIMINARIES}

Let us first recall some definition of some canstants:

$$
\begin{aligned}
& C_{\mathrm{NJ}}(X)=\sup _{\substack{\|, y \in X\\
\| x\|+\| y \|>0}} \frac{\|x+y\|^{2}+\|x-y\|^{2}}{2\left(\|x\|^{2}+\|y\|^{2}\right)}, \\
& C_{Z}(X)=\sup _{\substack{x, y \in X \\
\|x\|+\|y\|>0}} \frac{\|x+y\|\|x-z\|}{\|x\|^{2}+\|y\|^{2}}, \\
& C_{p}(X)=\sup _{\substack{x, y, z \in X \backslash\{0\} \\
x \neq y \neq z \neq x}} \frac{\|x-y\|\|z\|}{\|x-z\| y\|+\| z-y\|\| x \|} .
\end{aligned}
$$


It is well known that $1 \leqslant C_{p}(X) \leqslant 2$ for all normed spaces $X$. The Ptolemy inequality shows that $C_{p}(H)=1$ whenever $(H,\|\|$.$) is an inner product$ space. From the above definition, we can get that $C_{Z}(X) \leqslant C_{p}(X)$. However, there is no relationship between the Ptolemy constant $C_{p}(X)$ and von Neumann-Jordan constants $C_{N J}(X)^{7}$.

Recall that a norm on $\mathbb{R}^{2}$ is called absolute if $\|(z, w)\|=\|(|z|,|w|)\|$ for all $z, w \in \mathbb{R}$ and normalized if $\|(1,0)\|=\|(0,1)\|=1$. Let $N_{\alpha}$ denote the family of all absolute normalized norms on $\mathbb{R}^{2}$, and let $\Psi$ denote the family of all continuous convex functions on $[0,1]$ such that $\psi(1)=\psi(0)=1$ and $\max \{1-t, t\} \leqslant \psi(t) \leqslant 1(0 \leqslant t \leqslant 1)$. If $\psi(t)=$ $\psi(1-t)$ for all $t \in[0,1]$, we call the function $\psi$ is symmetric. It has been shown that $N_{\alpha}$ and $\Psi$ are a one-to-one correspondence in view of the following Proposition ${ }^{21}$.

Proposition 1 If $\|\cdot\| \in N_{\alpha}$ then $\psi(t)=\|(1-t, t)\| \in$ $\Psi$. On the other hand, if $\psi(t) \in \Psi$, define a norm $\|\cdot\|_{\psi}$ as

$$
\|(z, \omega)\|= \begin{cases}(|z|+|\omega|) \psi\left(\frac{|\omega|}{|z|+|\omega|}\right) & (z, \omega) \neq(0,0), \\ 0 & (z, \omega)=(0,0),\end{cases}
$$

then the norm $\|\cdot\|_{\psi} \in N_{\alpha}$.

A simple example of absolute normalized norm is usual $\ell_{p}(1 \leqslant p \leqslant \infty)$ norm. From Proposition 1 , one can easily get the corresponding function of the $\ell_{p}$ norm:

$$
\psi_{p}(t)= \begin{cases}\left\{(1-t)^{p}+t^{p}\right\}^{1 / p} & 1 \leqslant p<\infty \\ \max \{1-t, t\} & p=\infty\end{cases}
$$

Also, the above correspondence enable us to get many non- $\ell_{p}$ norms on $\mathbb{R}^{2}$. One of the properties of these norms is stated in the following result.

Proposition 2 Let $\psi, \varphi \in \Psi$ and $\varphi \leqslant \psi$. Put $M=$ $\max _{0 \leqslant t \leqslant 1} \frac{\psi(t)}{\varphi(t)}$, then

$$
\|\cdot\|_{\varphi} \leqslant\|\cdot\|_{\psi} \leqslant M\|\cdot\|_{\varphi} .
$$

Let $0<\omega<1$ and $1 \leqslant q<\infty$. The two-dimensional Lorentz sequence space $d^{(2)}(\omega, q)$ is $\mathbb{R}^{2}$ with the norm

$$
\|(x, y)\|_{\omega, q}=\left(\left(x^{*}\right)^{q}+\omega\left(y^{*}\right)^{q}\right)^{1 / q},
$$

where $\left(x^{*}, y^{*}\right)$ is the rearrangement of $(|x|,|y|)$ satisfying $x^{*} \geqslant y^{*}$, then $\|(x, y)\|_{\omega, q}$ is a symmetric absolute normalized norm on $\mathbb{R}^{2}$, and the corresponding convex function is ${ }^{16}$

$$
\psi_{\omega, q}(t) \begin{cases}\left((1-t)^{q}+\omega t^{q}\right)^{1 / q} & \text { if } 0 \leqslant t \leqslant 1 / 2 \\ \left(t^{q}+\omega(1-t)^{q}\right)^{1 / q} & \text { if } 1 / 2 \leqslant t \leqslant 1\end{cases}
$$

The authors get the dual norm of $\|(x, y)\|_{\omega, q}$ and the corresponding convex function ${ }^{22}$.

Theorem 1 Let $0<\omega<1$ and $1 \leqslant q<\infty$.

(i) If $q=1$, then

$$
\psi_{\omega, 1}^{*}(t)= \begin{cases}1-t & \text { if } 0 \leqslant t<\frac{\omega}{1+\omega}, \\ \frac{1}{1+\omega} & \text { if } \frac{\omega}{1+\omega} \leqslant t<\frac{1}{1+\omega}, \\ t & \text { if } \frac{1}{1+\omega} \leqslant t \leqslant 1 .\end{cases}
$$

(ii) If $1<q<\infty$, then

$$
\psi_{\omega, q}^{*}(t) \begin{cases}\left((1-t)^{p}+\omega^{1-p} t^{p}\right)^{\frac{1}{p}} & \text { if } 0 \leqslant t<\frac{\omega}{1+\omega}, \\ (1+\omega)^{\frac{1}{p}-1} & \text { if } \frac{\omega}{1+\omega} \leqslant t<\frac{1}{1+\omega}, \\ \left(t^{p}+\omega^{1-p}(1-t)^{p}\right)^{\frac{1}{p}} & \text { if } \frac{1}{1+\omega} \leqslant t \leqslant 1 .\end{cases}
$$

where $\frac{1}{p}+\frac{1}{q}=1$.

Now, let us put

$$
M_{1}=\max _{0 \leqslant t \leqslant 1} \frac{\psi_{2}(t)}{\psi(t)} \text { and } M_{2}=\max _{0 \leqslant t \leqslant 1} \frac{\psi(t)}{\psi_{2}(t)} .
$$

We consider the Ptolemy constant $C_{p}(X)$ of absolute normalized norms on $\mathbb{R}^{2}$, the exact values of $C_{p}(X)$ were also calculated in some classical Banach spaces ${ }^{9}$.

Theorem 2 Let $\psi \in \Psi$ and $\psi \geqslant \psi_{2}$, then $C_{p}\left(\|\cdot\|_{\psi}\right)=$ $M_{2}^{2}$.

Theorem 3 Let $\psi \in \Psi$ and $\psi \leqslant \psi_{2}$, if there exist $s, t \in[0,1](s<t)$ satisfying one of the following conditions:

(i) $\psi(s)=\psi_{2}(s), \psi(t)=\psi_{2}(t)$, and if we put $r=$ $\frac{\psi(s) t+\psi(t) s}{\psi(s)+\psi(t)}$, then $\frac{\psi_{2}(r)}{\psi(r)}=\frac{\psi(1-r)}{\psi_{2}(1-r)}=M_{1}$.

(ii) $\psi(s)=\psi_{2}(s), \psi(t)=\psi_{2}(t)$, and if we put $r=$ $\frac{\psi(t) s+\psi(s) t}{\psi(t)+\psi(s)(2 t-1)}$, then $\frac{\psi_{2}(r)}{\psi(r)}=\frac{\psi(1-r)}{\psi_{2}(1-r)}=M_{1}$. then $C_{p}\left(\|\cdot\|_{\psi}\right)=M_{1}^{2}$

Theorem 4 Let $\psi \in \Psi$ and $\psi(t)=\psi(1-t)$ for all $t \in[0,1]$. If $M_{1}=\frac{\psi_{2}(1 / 2)}{\psi(1 / 2)}$, then $C_{p}\left(\|\cdot\|_{\psi}\right)=M_{1}^{2} M_{2}^{2}$.

Theorem 5 Let $\psi \in \Psi$ and $\psi(t)=\psi(1-t)$ for all $t \in[0,1]$. If $M_{2}=\frac{\psi(1 / 2)}{\psi_{2}(1 / 2)}, M_{1}>1$ and if there exist $s, t \in[0,1](s<t)$ satisfying one of the following conditions: 
(i) $\psi_{2}(s)=M_{1} \psi(s), \psi_{2}(t)=M_{1} \psi(t)$, and if we put $r=\frac{\psi(s) t+\psi(t) s}{\psi(s)+\psi(t)}$, then $\psi(r)=M_{2} \psi_{2}(r)$.

(ii) $\psi_{2}(s)=M_{1} \psi(s), \psi_{2}(t)=M_{1} \psi(t)$, and if we put $r=\frac{\psi(t) s+\psi(s) t}{\psi(t)+\psi(s)(2 t-1)}$, then $\psi(r)=M_{2} \psi_{2}(r)$, then $C_{p}\left(\|\cdot\|_{\psi}\right)=M_{1}^{2} M_{2}^{2}$.

\section{MAIN RESULTS AND SOME EXAMPLES}

In this section, we will consider some new conditions of the convex function $\psi$ that Ptolemy constant $C_{p}(\|\cdot\|)$ coincide with the upper bound $M_{1}^{2} M_{2}^{2}$. For a norm $\|\cdot\|$ on $\mathbb{R}^{2}$, we write $C_{p}\left(\|\cdot\|_{\psi}\right)$ for $C_{p}\left(\mathbb{R}^{2},\|\cdot\|_{\psi}\right)$.

Theorem 6 Let $\psi \in \Psi$ and $\psi(t)=\psi(1-t)$ for all $t \in[0,1]$. If there exist unique points $t_{1}, t_{2} \in[0,1 / 2]$ such that

$M_{1}=\frac{\psi_{2}\left(t_{1}\right)}{\psi\left(t_{1}\right)}, M_{2}=\frac{\psi\left(t_{2}\right)}{\psi_{2}\left(t_{2}\right)}$ and $\left(1-t_{1}\right)\left(1-t_{2}\right)=\frac{1}{2}$,

then $C_{p}\left(\|\cdot\|_{\psi}\right)=M_{1}^{2} M_{2}^{2}$.

Proof: By Proposition 2, we have $\frac{1}{M_{1}}\|\cdot\|_{2} \leqslant\|\cdot\|_{\psi} \leqslant$ $M_{2}\|\cdot\|_{2}$. Let $x, y \in X,(x, y) \neq(0,0)$, where $X=\mathbb{R}^{2}$. Then

$$
\begin{aligned}
& \frac{\|x-y\|_{\psi}\|z\|_{\psi}}{\|x-z\|_{\psi}\|y\|_{\psi}+\|z-y\|_{\psi}\|x\|_{\psi}} \\
& \leqslant \frac{M_{2}^{2}\|x-y\|_{2}\|z\|_{2}}{\left(1 / M_{1}^{2}\right)\left(\|x-z\|_{2}\|y\|_{2}+\|z-y\|_{2}\|x\|_{2}\right)}, \\
& \leqslant M_{1}^{2} M_{2}^{2} C_{p}\left(\|.\|_{2}\right), \\
& =M_{1}^{2} M_{2}^{2} .
\end{aligned}
$$

From the definition of $C_{p}(X)$, implies that

$$
C_{p}\left(\|\cdot\|_{\psi}\right) \leqslant M_{1}^{2} M_{2}^{2} .
$$

On the other hand, note that $\left(1-t_{1}\right)\left(1-t_{2}\right)=\frac{1}{2}$, put $x=\left(1-t_{1}, t_{1}\right), y=\left(t_{1}, t_{1}-1\right), z=\left(1,2 t_{1}-1\right)$, then $x-y=\left(1-2 t_{1}, 1\right), x-z=\left(-t_{1}, 1-t_{1}\right)$ and $z-y=\left(1-t_{1}, t_{1}\right)$

$$
\begin{aligned}
\|x\|_{\psi} & =\psi\left(t_{1}\right)=\frac{\psi_{2}\left(t_{1}\right)}{M_{1}}, \\
\|y\|_{\psi} & =\psi\left(1-t_{1}\right)=\frac{\psi_{2}\left(t_{1}\right)}{M_{1}}, \\
\|z\|_{\psi} & =\frac{\psi\left(t_{2}\right)}{\left(1-t_{2}\right)}=\frac{M_{2} \psi_{2}\left(t_{2}\right)}{\left(1-t_{2}\right)}, \\
\|x-y\|_{\psi} & =\left(2-2 t_{1}\right) \psi\left(\frac{1}{2-2 t_{1}}\right)=\frac{\psi\left(1-t_{2}\right)}{\left(1-t_{2}\right)}, \\
& =\frac{M_{2} \psi_{2}\left(t_{2}\right)}{\left(1-t_{2}\right)},
\end{aligned}
$$

$$
\begin{aligned}
& \|x-z\|_{\psi}=\psi\left(1-t_{1}\right)=\frac{\psi_{2}\left(t_{1}\right)}{M_{1}}, \\
& \|z-y\|_{\psi}=\psi\left(t_{1}\right)=\frac{\psi_{2}\left(t_{1}\right)}{M_{1}},
\end{aligned}
$$

Since

$$
\sqrt{2}(1-t) \psi_{2}\left(\frac{1}{2-2 t}\right)=\psi_{2}(t) .
$$

Consequently, we have

$$
\begin{aligned}
\frac{\|x-y\|_{\psi}\|z\|_{\psi}}{\|x-z\|_{\psi}\|y\|_{\psi}+\|z-y\|_{\psi}\|x\|_{\psi}} & =\frac{M_{1}^{2} M_{2}^{2} \psi_{2}^{2}\left(t_{2}\right)}{2\left(1-t_{2}\right)^{2} \psi_{2}^{2}\left(t_{1}\right)} \\
& =M_{1}^{2} M_{2}^{2} .
\end{aligned}
$$

From the inequality (1) and the above equality, then $C_{p}\left(\|\cdot\|_{\psi}\right)=M_{1}^{2} M_{2}^{2}$. This completes the proof.

Remark 1 When the function $\psi$ is symmetric, if $t_{1}=1 / 2$, from the equality $\left(1-t_{1}\right)\left(1-t_{2}\right)=\frac{1}{2}$ in Theorem 6 , then $t_{2}=0, M_{2}=1$, we can also obtain the Ptolemy constant $C_{p}(\|\|$.$) coincide with$ the upper bound $M_{1}^{2} M_{2}^{2}$ by Theorem 4. If $t_{2}=1 / 2$, then $t_{1}=0, M_{1}=1$ and $\psi \geqslant \psi_{2}$, we can also obtain the Ptolemy constant $C_{p}(\|\cdot\|)$ coincide with the upper bound $M_{1}^{2} M_{2}^{2}$ by Theorem 2. However, $M_{1}=$ $\frac{\psi_{2}(t)}{\psi(t)}$ and $M_{2}=\frac{\psi(t)}{\psi_{2}(t)}$ need not attain the maximum at $t=1 / 2$ in Theorem 6 . Indeed, there are some examples satisfy this case ${ }^{9}$. Moreover, we give a Example 1 to satisfy the condition in Theorem 6.

Example 1 Let $0 \leqslant c \leqslant 1$, the corresponding convex function is given by

$$
\psi_{c}=\max \left\{1-c t, 1-c+c t, 1-\frac{c^{2}}{2}\right\}(0 \leqslant t \leqslant 1) .
$$

Then

(i) If $0 \leqslant c \leqslant-1+\sqrt{3}$, then $C_{p}\left(\|\cdot\|_{\psi_{c}}\right)=\frac{\left(2-c^{2}\right)^{2}}{2}$.

(ii) If $-1+\sqrt{3}<c \leqslant 1$, then $C_{p}\left(\|\cdot\|_{\psi_{c}}\right)=\frac{2\left(c^{2}-2 c+2\right)^{2}}{\left(2-c^{2}\right)^{2}}$.

Proof: If $0 \leqslant c \leqslant-1+\sqrt{3}$, then $\psi \geqslant \psi_{2}$. From Theorem 2, then

$$
C_{p}\left(\|\cdot\|_{\psi}\right)=M_{2}^{2}=\frac{\left(2-c^{2}\right)^{2}}{2} .
$$

If $-1+\sqrt{3}<c \leqslant 1$, then

$$
\begin{aligned}
& M_{1}^{2}=\frac{\psi_{2}^{2}\left(t_{1}\right)}{\psi_{c}^{2}\left(t_{1}\right)}=\frac{2\left(c^{2}-2 c+2\right)^{2}}{\left(2-c^{2}\right)}, \\
& M_{2}^{2}=\frac{\psi_{c}^{2}\left(t_{2}\right)}{\psi_{2}^{2}\left(t_{2}\right)}=c^{2}-2 c+2,
\end{aligned}
$$


where $t_{1}=c / 2, t_{2}=(1-c) /(2-c)$, they satisfy (1$\left.t_{1}\right)\left(1-t_{2}\right)=\frac{1}{2}$. From Theorem 6 , then $C_{p}\left(\|\cdot\|_{\psi}\right)=$ $M_{1}^{2} M_{2}^{2}=\frac{2\left(c^{2}-2 c+2\right)^{2}}{\left(2-c^{2}\right)^{2}}$.

The exact values of $C_{p}\left(d^{(2)}(\omega, 2)\right)$ were calculated by Theorem 3 . In the following, we completely compute the Ptolemy constant of two-dimensional Lorentz sequence spaces $d^{(2)}(\omega, q)(1 \leqslant q<\infty)$ and their dual spaces $d^{(2)}(\omega, q)^{*}$ in the case of $q=1$ and $q \geqslant 2$.

Theorem 7 Let $0<\omega<1$ and $1 \leqslant q<\infty$. The twodimensional Lorentz sequence space $d^{(2)}(\omega, q)$ is $\mathbb{R}^{2}$ with the norm

$$
\|(x, y)\|_{\omega, q}=\left(\left(x^{*}\right)^{q}+\omega\left(y^{*}\right)^{q}\right)^{1 / q},
$$

where $\left(x^{*}, y^{*}\right)$ is the rearrangement of $(|x|,|y|)$ satisfying $x^{*} \geqslant y^{*}$.

(i) If $1 \leqslant q<2$ then

$$
C_{p}(\|\cdot\|)=\left[1+\omega^{2 /(2-q)}\right]^{2 / q-1} \max \left\{\frac{2}{(1+\omega)^{2 / q}}, 1\right\} .
$$

(ii) If $q \geqslant 2$ then $C_{p}(\|\|)=.2\left(\frac{1}{1+\omega}\right)^{2 / q}$.

Proof: If $1 \leqslant q<2$, we get the following inequality ${ }^{15}$ :

$$
a\|x\|_{2} \leqslant\|x\|_{\omega, q} \leqslant b\|x\|_{2} .
$$

where

$$
a=\min \left\{\frac{(1+\omega)^{\frac{1}{q}}}{\sqrt{2}}, 1\right\} \quad \text { and } \quad b=\left[1+\omega^{\frac{2}{2-q}}\right]^{\frac{1}{q}-\frac{1}{2}} .
$$

Let $x, y \in X,(x, y) \neq(0,0)$, where $X=\mathbb{R}^{2}$. Then

$$
\begin{aligned}
& \frac{\|x-y\|_{\omega, q}\|z\|_{\omega, q}}{\|x-z\|_{\omega, q}\|y\|_{\omega, q}+\|z-y\|_{\omega, q}\|x\|_{\omega, q}} \\
& \leqslant \frac{b^{2}\left(\|x-y\|_{2}\|z\|_{2}\right)}{a^{2}\left(\|x-z\|_{2}\|y\|_{2}+\|z-y\|_{2}\|x\|_{2}\right)}, \\
& \leqslant \frac{b^{2}}{a^{2}} C_{p}\left(\|\cdot\|_{2}\right)=\frac{b^{2}}{a^{2}} .
\end{aligned}
$$

From the definition of $C_{p}(X)$, implies that

$$
C_{p}\left(d^{(2)}(\omega, q)\right) \leqslant \frac{b^{2}}{a^{2}} .
$$

On the other hand, take $x=(1,0), y=$ $\left(0, \omega^{1 /(2-q)}\right)$, and $z=\left(1, \omega^{1 /(2-q)}\right)$, then

$C_{p}\left(d^{(2)}(\omega, q)\right)$

$\geqslant \frac{\|x-y\|_{\omega, q}\|z\|_{\omega, q}}{\|x-z\|_{\omega, q}\|y\|_{\omega, q}+\|z-y\|_{\omega, q}\|x\|_{\omega, q}}$,

$$
\begin{aligned}
& =\frac{\left\|\left(1,-\omega^{1 /(2-q)}\right)\right\|_{\omega, q}\left\|\left(1, \omega^{1 /(2-q)}\right)\right\|_{\omega, q}}{\left\|\left(0,-\omega^{1 /(2-q)}\right)\right\|_{\omega, q}\left\|\left(0, \omega^{1 /(2-q)}\right)\right\|_{\omega, q}+\|(1,0)\|_{\omega, q}^{2}}, \\
& =\frac{\left(1+\omega^{2 /(2-q)}\right)^{2 / q}}{1+\omega^{2 /(2-q)}}=b^{2} .
\end{aligned}
$$

Also, let us put $x=\left(1+\omega^{1 /(2-q)}\right)(1,1), y=(1+$ $\left.\omega^{1 /(2-q)}\right)(1,-1)$, and $z=\left(2,2 \omega^{1 /(2-q)}\right)$, so

$$
\begin{aligned}
& C_{p}\left(d^{(2)}(\omega, q)\right) \geqslant \frac{\|x-y\|_{\omega, q}\|z\|_{\omega, q}}{\|x-z\|_{\omega, q}\|y\|_{\omega, q}+\|z-y\|_{\omega, q}\|x\|_{\omega, q}} \\
& =\frac{4\left[1+\omega \omega^{q /(2-q)}\right]^{2 / q}}{(1+\omega)^{2 / q}\left[\left(1+\omega^{1 /(2-q)}\right)^{2}+\left(1-\omega^{1 /(2-q)}\right)^{2}\right]}, \\
& =\frac{2\left(1+\omega^{2 /(2-q)}\right)^{2 / q-1}}{(1+\omega)^{2 / q}}=b^{2} \frac{2}{(1+\omega)^{2 / q}} \text {. }
\end{aligned}
$$

From the above discussion, we have the equality (i).

If $2 \leqslant q<\infty$, then

$$
\frac{(1+\omega)^{1 / q}}{\sqrt{2}}\|x\|_{2} \leqslant\|x\|_{\omega, q} \leqslant\|x\|_{2} .
$$

From the above inequality, we can similarly have

$$
C_{p}\left(d^{(2)}(\omega, q)\right) \leqslant 2\left(\frac{1}{1+\omega}\right)^{2 / q} .
$$

On the other hand, take $x=(a, a), y=(a,-a)$, and $z=(2 a, 0)$, where $a=\frac{1}{(1+\omega)^{1 / q}}$, then $\|x\|_{\omega, q}=$ $\|y\|_{\omega, q}=1$, and so

$$
\begin{aligned}
C_{p}\left(d^{(2)}(\omega, q)\right) & \|x-y\|_{\omega, q}\|z\|_{\omega, q} \\
& \geqslant \frac{\|x-z\|_{\omega, q}\|y\|_{\omega, q}+\|z-y\|_{\omega, q}\|x\|_{\omega, q}}{\| 2} \\
& =\frac{(2 a)^{2}+(2 a)^{2}}{4}=2\left(\frac{1}{1+\omega}\right)^{2 / q} .
\end{aligned}
$$

From the inequality (3), we have (ii).

In order to get the exact value of $C_{p}\left(d^{(2)}(\omega, q)^{*}\right)$ in the case of $q=1$, we need the following example $^{9}$.

Example 2 Let $1 / 2 \leqslant \beta \leqslant 1$, the two-dimensional space $X_{\beta}$ which has corresponding convex function is given by $\psi_{\beta}(t)=\max \{1-t, t, \beta\}$. Then

(i) If $1 / 2 \leqslant \beta \leqslant 1 / \sqrt{2}$, then $C_{p}\left(\|\cdot\|_{\psi_{\beta}}\right)=\frac{\beta^{2}+(1-\beta)^{2}}{\beta^{2}}$.

(ii) If $1 / \sqrt{2}<\beta \leqslant 1$, then $C_{p}\left(\|\cdot\|_{\psi_{\beta}}\right)=2\left(\beta^{2}+(1-\right.$ $\beta)^{2}$ ).

Theorem 8 Let $0<\omega<1$ and $1 / p+1 / q=1$, where $\psi_{\omega, q}^{*}(t)$ is the corresponding convex function to dual norm of $\|(x, y)\|_{\omega, q}$. 
(i) If $q=1$ then

$$
C_{p}\left(d^{(2)}(\omega, q)^{*}\right)=\left\{\begin{array}{ll}
\frac{2\left(1+\omega^{2}\right)}{(1+\omega)^{2}} & \text { if } 0<\omega \leqslant \sqrt{2}-1, \\
1+\omega^{2} & \text { if } \sqrt{2}-1<\omega<1 .
\end{array} .\right.
$$

(ii) If $q \geqslant 2$ then $C_{p}\left(d^{(2)}(\omega, q)^{*}\right)=2\left(\frac{1}{1+\omega}\right)^{2 / q}$.

Proof: By the definition of $\psi_{\beta}(t)=\max \{1-t, t, \beta\}$, we have

$$
\psi_{\beta}(t)= \begin{cases}1-t & \text { if } 0 \leqslant t<1-\beta \\ \beta & \text { if } 1-\beta \leqslant t<\beta \\ t & \text { if } \beta \leqslant t \leqslant 1\end{cases}
$$

If $q=1$, let $\beta=\frac{1}{\omega}$, then $\psi_{\omega, 1}^{*}(t)=\psi_{\beta}(t)$. From Example 2, we obtain the following conclusion (i):

$$
C_{p}\left(d^{(2)}(\omega, q)^{*}\right)= \begin{cases}\frac{2\left(1+\omega^{2}\right)}{(1+\omega)^{2}} & \text { if } 0<\omega \leqslant \sqrt{2}-1, \\ 1+\omega^{2} & \text { if } \sqrt{2}-1<\omega<1 .\end{cases}
$$

If $q \geqslant 2$, from the the symmetry of $\psi_{\omega, q}^{*}(t)$, we can only consider the function $g(t)=\frac{\psi_{\omega, q}^{*}(t)}{\psi_{2}(t)}$ from $[0,1 / 2]$ into $\mathbb{R}$. The derivative of $g(t)$ at $t \in\left(0, \frac{\omega}{1+\omega}\right)$ is

$$
\begin{aligned}
g^{\prime}(t)=\frac{\left((1-t)^{p}+\omega^{1-p} t^{p}\right)^{1 / p-1}}{} & \left((1-t)^{2}+t^{2}\right)^{3 / 2} \\
& \times\left(-t(1-t)^{p-1}+\omega^{1-p}(1-t) t^{p-1}\right) .
\end{aligned}
$$

Since $q \geqslant 2$, then $1 \leqslant p \leqslant 2$, we have $g^{\prime}(t) \geqslant 0$ for all $t \in\left(0, \frac{\omega}{1+\omega}\right)$, so $g(t)$ is non-decreasing on $\left[0, \frac{\omega}{1+\omega}\right]$. Moreover, it is obvious that $g(t)$ is non-decreasing on $\left[\frac{\omega}{1+\omega}, \frac{1}{2}\right]$. Therefore $g(t)$ is non-decreasing on $\left[0, \frac{1}{2}\right]$, we obtain that $\frac{\psi_{\omega, q}^{*}(t)}{\psi_{2}(t)}$ has the maximum $M_{2}=$ $\frac{2\left(\frac{1}{1+\omega}\right)^{1 / q}}{\sqrt{2}}>1$ at $t=1 / 2$. Thus by Theorem 2 , we get

$$
C_{p}\left(d^{(2)}(\omega, q)^{*}\right)=M_{2}^{2}=2\left(\frac{1}{1+\omega}\right)^{2 / q} .
$$

Remark 2 From Theorem 7 and Theorem 8, $C_{p}\left(d^{(2)}(\omega, q)\right)=C_{p}\left(d^{(2)}(\omega, q)^{*}\right)$ in the case of $q=1$ and $q \geqslant 2$. Although we do not know the exact value of $C_{p}\left(d^{(2)}(\omega, q)^{*}\right)$ in the case of $1<q<2$, It will be interesting to determine if the equality $C_{p}(X)=C_{p}\left(X^{*}\right)$ holds for any Banach space $X$.

Acknowledgements: The work was supported by the Scientific and Technological Research Program of Chongqing Municipal Education Commission (Grant No. KJQN201801205), the Chongqing New-star Plan of Science and Technology, the Scientific Technological
Research Program of the Chongqing Three Gorges University (No. 16PY11), the Chongqing Municipal Key Laboratory of Institutions of Higher Education (Grant No. [2017] 3), the Program of Chongqing Development and Reform Commission (Grant No.2017[1007]), the Key Laboratory for Nonlinear Science and System Structure, Chongqing Three Gorges University.

\section{REFERENCES}

1. Ikeda T, Kato M (2014) Notes on von NeumannJordan and James constants for absolute norms on $\mathbb{R}^{2}$. Mediterr. J. Math. 11, 633-42.

2. Kato M, Maligranda L, Takahashi Y (2001) On James and Jordan-von Neumann constants and normal structure coefficient of Banach spaces. Studia Math. 144, 275-95.

3. Saito K, Kato M, Takahashi Y (2000) Von NeumannJordan constant of absolute normalized norms on $C^{2}$. J. Math. Anal. Appl. 244, 515-32.

4. Alonso J, Martin P (2006) A counterexample for a conjecture of G. Zbăganu about the NeumannJordan constant. Rev. Roum. Math. Pures Appl. 51, $135-41$.

5. Mizuguchi H, Saito K (2011) Some geometric constants of absolute normalized norms on $\mathbb{R}^{2}$. Ann. Funct. Anal. 2, 22-33.

6. Zbăganu G (2001) An inequality of M. Rădulescu and S. Rădulescu which characterizes inner product spaces. Rev. Roumaine Math. Pures Appl. 47, 253-7.

7. Llorens-Fuster E, Mazcuñán-Navarro E, Reich $S$ (2010) The Ptolemy and Zbăganu constants of normed spaces. Nonlinear Analysis. 72, 3984-93.

8. Pinchover Y, Reich S, Shafrir I (2001) The Ptolemy constant of a normed space. Amer. Math. Monthly. 108, 475-6.

9. Zuo Z (2015) A reconsideration on the Ptolemy Constant of absolute normalized norms. Acta Mathematica Sinica(Chinese Series). 58, 337-44.

10. Jiménez-Melado A, Llorens-Fuster E, Saejung S (2006) The von Newman-Jordan constant, weak orthogonality and normal structure in Banach spaces. Proc. Am. Math. Soc. 134, 355-64.

11. Saejung S (2006) On James and von NeumannJordan constants and sufficient conditions for the fixed point property. J. Math. Anal. Appl. 323, 101824.

12. Zuo Z, Cui Y (2009) Some modulus and normal structure in Banach space. Journal of Inequalities and Applications. 2009, Article ID 676373.

13. Pinchover Y (1999) Maximum and anti-maximum principles and eigenfunctions estimates via perturbation theory of positive solutions of elliptic equations. Math. Ann. 314, 555-90.

14. Kalton N, Verbitsky I (1999) Nonlinear equations and weighted norm inequalities. Trans. Amer. Math. Soc. 351, 3441-97. 
15. Kato M, Maligranda L (2001) On James and von Neumann-Jordan constants of Lorentz sequence spaces. J. Math. Anal. Appl. 258, 457-65.

16. Mitani K, Saito K (2003) The James constant of absolute norms on $\mathbb{R}^{2}$. J. Nonlinear Convex Anal. 4, 399-410.

17. Mizuguchi H, Saito K, Tanaka R (2014) The DunklWilliams constant of symmetric octagonal norms on $\mathbb{R}^{2}$ II. Nihonkai Math. J. 25, 151-72.

18. Zuo Z (2012) The Ptolemy constant of absolute normalized norms on $\mathbb{R}^{2}$. Journal of Inequalities and Applications. 2012, 1-9.

19. Zuo Z, Tang C (2016) Jordan-von Neumann type constant and fixed points for multivalued nonexpansive mappings. Journal of Mathematical Inequalities. 10, 649-57.

20. Mizuguchi H, Saito K (2016) On the upper bound of some geometric constants of absolute normalized norms on $\mathbb{R}^{2}$. Mediterr. J. Math. 13, 309-22.

21. Bonsall F, Duncan J (1973) Numerical Ranges II, London Mathematical Society Lecture Notes Series, vol 10, Cambridge Univ. Press.

22. Mitani K, Saito K (2009) Dual of two dimensional Lorentz sequence spaces. Nonlinear Anal. 71, 523847. 